\title{
Evaluation of Cardiovascular Autonomic Function in Prediabetics: Need of Tomorrow
}

\section{IJCRR \\ Section: Healthcare \\ ISI Impact Factor \\ (2019-20): 1.628 \\ IC Value (2019): 90.81 \\ $\operatorname{SJIF}(2020)=7.893$}

(c) (i) (8)

Copyright@IJCRR

\section{Amit Baheti ${ }^{1}$, Rajesh Sarode ${ }^{2}$, Sunil Kumar ${ }^{2 *}$, Sourya Acharya ${ }^{2}$, Anil Wanjari², Sachin Agrawal2, Shilpa Bawankule²}

'Senior Resident, Department of Medicine, Jawaharlal Nehru Medical College, Datta Meghe Institute of Medical Science (Deemed to be University) Sawangi (Meghe), Wardha, Maharashtra, India; ${ }^{2}$ Professor, Department of Medicine, Jawaharlal Nehru Medical College, Datta Meghe Institute of Medical Science (Deemed to be University) Sawangi (Meghe), Wardha, Maharashtra, India.

\section{ABSTRACT}

Introduction: Cardiovascular autonomic neuropathy (CAN) is a chronic complication of diabetes with life-threatening complications ranging from resting tachycardia to sudden death. Considering hyperglycaemia as the main etiopathogenic factor of $\mathrm{CAN}$, its detection in the prediabetic stage will have a huge impact in delaying the development of severe forms of CAN and its complications.

Objective: This study had been planned to evaluate cardiac autonomic functions in prediabetics by cardiac autonomic reflex tests.

Method: This cross-sectional study was carried out in individuals with newly detected prediabetics and normoglycemic controls attending the medicine outpatient department for routine checkups. Anthropometric indices, blood pressure and serum lipids were assessed. Cardiac autonomic reflex tests were performed and individuals were divided according to Ewing's criteria of CAN. The cardiovascular risk factors were compared to see their association with CAN.

Result: In this study CAN was found in $15.2 \%$ prediabetics and $3.6 \%$ normoglycaemic age and gender-matched controls $(p=<0.0001, S)$. No significant association of CAN with cardiovascular risk factors like Body Mass Index, waist-hip ratio, hypertension and serum lipids was found.

Conclusion: As per multivariate regression analysis results, prediabetic status was considered to be the sole risk factor for the presence of CAN.

Key Words: Autonomic reflex test, Cardiovascular autonomic neuropathy, Prediabetes, Diabetes, Risk factors, Anthropometric indices

\section{INTRODUCTION}

Cardiac autonomic neuropathy (CAN) is a chronic complication of diabetes with significant life-threatening effects such as resting tachycardia, reduced exercise tolerance, orthostatic hypotension, silent myocardial ischemia and infarction, arrhythmias, perioperative cardiovascular instability, cardiomyopathy, sudden death. ${ }^{1} \mathrm{CAN}$ is caused by the impairment of the autonomic nerve fibres regulating heart rate, myocardial contractility, cardiac electrophysiology, blood vessel constriction and dilatation. The exact pathogenesis of CAN is still less understood. ${ }^{1,2}$ Many of the proposed mechanisms include hyperglycemia, autoimmunity, genetics and inflammation leading to neuronal degeneration, mitochondrial dysfunction and formation of reactive oxygen species (ROS). These factors, in particular, will decline the cardiac nerve function and thus hyperglycemia, may be considered as the main pathogenic determinant in early cardiac autonomic dysfunction. ${ }^{1}$ Patients with CAN become symptomatic only in the later stages of the disease, i.e. in severe CAN with no specific treatment option being available at present. The reversal of cardiovascular autonomic neuropathy is thought to be possible in the early stages of the disease with adequate glycaemic control. ${ }^{2}$ Early diagnosis is the essential step in preventing the life-threatening complications of CAN. Out of the battery of tests available for diagnosis of CAN, Cardiac autonomic reflex tests (CART) are considered to be the gold standard, they are well standardized and non-invasive. CAN was classified into early CAN defined as the presence of one abnormal CART and severe CAN as the presence of orthostatic hypotension with two or more abnormal CART. ${ }^{3}$

Corresponding Author:

Sunil Kumar, Professor, Department of Medicine, Jawaharlal Nehru Medical College, Datta Meghe Institute of Medical Science (Deemed to be University) Sawangi (Meghe), Wardha, Maharashtra, India; Mobile: 9850393787; Email: sunilkumarmed@gmail.com

ISSN: $2231-2196$ (Print)

Received: 07.01 .2021
ISSN: 0975-5241 (Online)

Revised: 12.02 .2021
Published: 11.10 .2021 
Many studies are investigating cardiac autonomic dysfunction in people with diabetes, showing its prevalence ranging from $50-93 \% .^{1,2}$ This microvascular complication in people with diabetes is significant with uncontrolled diabetics, longer duration of diabetes and other microvascular complications like diabetic nephropathy and retinopathy. ${ }^{4}$ limited studies are evaluating CAN in prediabetics, of the various studies doing so its prevalence is found to be $10-20 \%{ }^{5,6}$

The current study was conducted to evaluate prediabetics for cardiovascular autonomic neuropathy by performing CART, its association with different cardiovascular risk factors like Body mass index, Waist-hip ratio, lipid profile and systemic hypertension and finding the prevalence of CAN by using Ewing's criteria.

\section{METHODS}

This prospective observational cross-sectional study was carried out in the department of medicine, at rural Medical College, in rural central India, from October 2017 to September 2019, after approval by the Institutional Ethics Committee with an approval letter numbered DMIMS (DU)/ IEC/2017-18/6761.

Subjects coming for routine evaluation and patient's relatives attending medicine OPD under 'Aadhar Yojana of Maharashtra Government (provide free of cost evaluation and treatment) were screened for prediabetes. Subjects with prediabetes as per WHO criteria i.e. fasting plasma glucose (FPG) of 6.1-6.9 mmol/L (110 to $125 \mathrm{mg} / \mathrm{dL}$ ) and $2 \mathrm{~h}$ plasma glucose of 7.8-11.0 mmol/L (140-200 mg/dL) after ingestion of $75 \mathrm{~g}$ of oral glucose load or a combination of the two based on a $2 \mathrm{~h}$ oral glucose tolerance test (OGTT) were divided into cases and age and gender-matched normoglycaemic were taken as control subjects. ${ }^{7}$ Selection of controls was taken from the subjects coming from the aadhar yojna and age and gender matching was done on a group basis.

Sample size formula based on prevalence ${ }^{5}$ was used: $\mathrm{N}=\mathrm{Z}$ $1-\alpha / 2^{2 *} p^{*}(1-\mathrm{P}) / \mathrm{d} 2$

We used $\mathrm{Z} 1-\alpha / 2=1.96, P=20 \%$, i.e. $0.2, d=5 \%$, i.e. 0.05 in the equation. So by applying the above formula, $N=1.96$ $2 * 0.2 *(1-0.2) /\left(0.05^{2}\right)=245.86$. Thus 250 subjects were taken in both case and control groups.

Both these groups were interviewed, examined, investigated in the study after informed written consent. Subjects with diabetes mellitus, acute coronary syndrome and ischemic heart disease, arrhythmias or taking anti-arrhythmic drug therapy, stroke, peripheral vascular diseases, chronic alcoholism, history of taking medications like antidepressants, beta-blockers and those who were unable to perform CARTs were excluded from the study shown in flow chart [figure 1].
All patients underwent a thorough anthropometric and physical examination, including measurement of body mass index (BMI), Waist Hip ratio, resting heart rate and blood pressure. The subjects were divided into different categories as per,(I) WHO Categories of Body Mass Index (BMI) for the Asia-Pacific Region into underweight $(<18.5 \mathrm{~kg} / \mathrm{m} 2)$, normal (18.5-22.99 kg/m2), overweight (23-27.49 kg/m2) and obese (27.5 kg/m2 and above),(II) WHO cut off values for the waist-hip ratio for males $-<0.9$ and Females $-<0.85$ were used and(III) those with the systolic Blood pressure of more than $140 \mathrm{~mm}$ of hg and diastolic Blood pressure of more than $90 \mathrm{~mm}$ of hg were defined as hypertensive. ${ }^{8}$

In all patients, two blood samples were collected once in the morning after an overnight fast and another after Oral Glucose Tolerance Test. Fasting blood glucose and Fasting lipid profile (Total cholesterol/TC, high-density lipoprotein/ HDL, triglycerides/TG) levels were measured with an automatic analyzer. Low-density lipoprotein/LDL and very-lowdensity lipoprotein/VLDL were measured indirectly; using Friedwald calculation.

Cardiac autonomic functions were evaluated with a cardiac autonomic analyzer (CAN win, Windows base Cardiac Autonomic Neuropathy Analysis system, version 1, Genesis Medical Systems). It analyses both sympathetic and parasympathetic autonomic nervous system functions. The system uses electrocardiograms and automatic non-invasive blood pressure monitoring to conduct a battery of five cardiac autonomic reflex tests (CART). ${ }^{3}$

After the interview and the anthropometric measurements, the subjects were explained in detail about the CARTs. The tests were performed on subjects with minimal, comfortable loose clothing, in a quiet ambient room with dim lighting and room temperature of $26-30^{\circ} \mathrm{C}$.

The following mentioned tests were performed sequentially as per the set computerized protocol, and the automated computerized result was used for the diagnosis of CAN. ${ }^{5}$

Tests reflecting parasympathetic dysfunction were a) Resting heart rate: A resting heart rate $>100$ beats per minute was considered abnormal. b) Heart rate variation during deep breathing: All patients were asked to lie supine and after 2 min of normal breathing, and then asked to breathe deeply at 6 breaths per minute. Heart rate was calculated on continuous ECG recording. The expiration: inspiration (E: I) ratio was calculated as the Mean value of the longest RR interval during expiration upon the Mean value of the shortest RR interval during deep inspiration. E: I ratio of $\geq 1.21$, 1.111.20 and $\leq 1.10$ were considered as normal, borderline and abnormal respectively.Heart rate response to standing (30:15 ratios): The patient was asked to lie supine quietly for $3 \mathrm{~min}$ and then asked to stand up. A continuous electrocardiogram (ECG) was recorded and the 30:15 ratios were calculated as, 
$\mathrm{RR}$ interval at $30^{\text {th }}$ beat after standing upon $\mathrm{RR}$ interval at $15^{\text {th }}$ beat after standing. 30:15 ratios of $\geq 1.04,1.01-1.03$ and $\leq 1.00$ were considered as normal, borderline and abnormal respectively.

Valsalva ratio: The patient was asked to blow into a mouthpiece connected to a manometer to keep the pressure up to 40 $\mathrm{mmHg}$ and to maintain it for $15 \mathrm{~s}$, while a continuous ECG recording was done. After $30 \mathrm{~s}$ ECG was monitored again for $15 \mathrm{~s}$. The Valsalva ratio was calculated as, Longest RR interval after the manoeuvre upon the Shortest RR interval during the manoeuvre. This procedure was avoided in patients with proliferative retinopathy. A Valsalva ratio of $\geq 1.21,1.11-1.20$ and $\leq 1.10$ were considered as normal, borderline and abnormal respectively.

Tests for sympathetic dysfunction were

1. Blood pressure (BP) response to standing: Patients were asked to stand in the supine position and remain standing for $2 \mathrm{~min}$. The postural fall after 2 minutes in $\mathrm{BP}$ was taken as the difference between systolic BP lying and the systolic BP standing. A decline in SBP by $\leq 11,11-29$ and $\geq 30 \mathrm{~mm}$ of mercury were considered as normal, borderline and abnormal respectively.

2. Blood pressure response to sustained handgrip: Handgrip dynamometer was applied on the dominant arm, and patients were asked to apply pressure on it three times. The maximum voluntary contraction was taken as the highest of three readings. All patients were instructed to maintain handgrip steadily at $30 \%$ of maximum contraction for at least 5 minutes. Blood pressure was taken on another arm at rest and the end of the grip. A rise of DBP by $\geq 16,11-15$ and $\leq 10 \mathrm{~mm}$ of hg were considered as normal, borderline and abnormal respectively.

The main outcome variable was Cardiac Autonomic Neuropathy (CAN) derived from E/I Ratio, Valsalva ratio and response to standing 30:15 (parasympathetic function), postural hypotension and blood pressure response to sustained handgrip (sympathetic function). As per the CART results, subjects were classified into different categories of CAN according to Ewing's criteria into normal, early, definite and severe CAN. ${ }^{3}$

\section{Data analysis:}

Categorical variables were presented in number and percentage (\%), and continuous variables were presented as mean \pm SD. Qualitative variables were correlated using the ChiSquare test/Fisher's exact test. Multivariate logistic regression was used to find out the risk factors of CAN. A P-value of $<0.05$ was considered statistically significant. Statistical Package for Social Sciences (SPSS) version 21.0.was used for data analysis.

\section{RESULTS}

In our study, we have enrolled 250 cases and 250controls with mean age having $43.91 \pm 10.83$ and $44.07 \pm 11.27$ years respectively. The mean BMI for cases was $23.94 \pm 3.64 \mathrm{~kg} /$ $\mathrm{m}^{2}$. The mean BMI for controls was $22.54 \pm 2.53 \mathrm{~kg} / \mathrm{m}^{2}$. The difference in BMI of cases and controls was statistically significant $(<0.0001, \mathrm{~S})$. The mean waist-hip ratio of cases was $0.92 \pm 0.06$ and in controls was 0.88 and SD of 0.07 , which was statistically significant $(<0.0001, \mathrm{~S})$. All other baseline characteristics are shown inTable 1.

Regarding the cardiac autonomic functions, 38 (15.2\%) cases and $9(3.6 \%)$ controls had CAN as per Ewing's criteria. Out of the subjects with CAN 28(11.2\%) cases and 9(3.6\%), controls had early CAN, 10(4\%) cases and no controls had definite CAN, and no case or control had a severe form of CAN as shown in figure 2. The difference in CAN in the two groups was found to be statistically significant $(<0.0001, \mathrm{~S})$. The results of individual CARTs were shown in Table 2, of the CARTs E: I ratio was found to be the most sensitive test.

Of the various parameters, blood sugar was the only significant parameter showing association with CAN as per the univariate regression analysis. No association was seen with age, sex, anthropometric parameters like BMI and W/H ratio, hypertension, biochemical parameters like lipid profile as shown in table 3 . This analysis was univariate as the only variable in this study was CAN and the other parameters were studied to look for associated confounding factors.

\section{DISCUSSION}

In our study, the Prevalence of CAN was more in the prediabetic group (15.2\%) as compared to the control group (3.6\%). Most of the subjects with CAN had an early form of CAN while no subject had a severe form of CAN. Our results were following studies conducted by Ziegler et al. where autonomic neuropathy was detected using Heart Rate Variability and Heart Rate Turbulence by Holter monitoring and was seen in $4.5 \%$ of subjects with Normal Glucose Tolerance(NGT) and $11.4 \%$ in subjects with combined Impaired Fasting Glucose-Impaired Glucose Tolerance(IFGIGT). ${ }^{6}$ Similarly in the study conducted byDimova et al. showed that CAN be detected by performing CARTs was found in $12.3 \%$ of NGT, $19.8 \%$ of prediabetes $(13.2 \%$ of IFG and $20.6 \%$ of IGT). ${ }^{5}$

Hyperglycaemia is the most likely pathogenetic factor for CAN which acts through the polyol pathway and causes direct neuronal damage and activation of protein kinase $\mathrm{c}$ leading to vasoconstriction and decreased neuronal blood flow thus altered nerve function. Others may be increased oxidative stress, increased free radical production, dysfunction of nitric oxide production, immune mechanisms, and neurotrophic 
growth factors deficiency which leads to nerve hypoxia and development of CAN. ${ }^{9}$

There are several reported risk factors associated with the development of CAN which includes age, duration of diabetes, glycemic control, the presence of other microvascular complications, hypertension, dyslipidemia (decreased HDL, increased LDL, and TGs levels), and obesity. ${ }^{10}$ In our study there was no significant association of CAN seen with these proposed risk factors.

The presence of CAN is strongly associated with increased mortality and morbidity like stroke, coronary artery disease and silent myocardial ischemia. This has been reflected by the results from the European Epidemiology and Prevention of Diabetes (EURODIAB) study and Action to Control Cardiovascular Risk in Diabetes (ACCORD) trial. ${ }^{11,12}$ Early diagnosis and prompt treatment is considered as the mainstay to prevent the development of severe forms of CAN and its complications, thereby detection of CAN in the prediabetic stage will be of great prognostic impact. Treatment of CAN includes symptomatic management and therapies to slow or reverse its progression. The treatment of CAN focuses on lifestyle changes, sugar control, antioxidants and medical therapy for orthostatic hypotension.

The limitation of our study is that this study was conducted using a hospital-based sample; therefore, further research using community-based studies and RCTs will be needed to confirm whether these conclusions can be generalized.

\section{CONCLUSION}

Prediabetics have increased cardiovascular risk factors, CAN being one of them which develops even before overt diabetes. Thence CAN detection should be advised in the workup of prediabetes, whether symptomatic or not along with measurement of other cardiovascular risk factors.

\section{ACKNOWLEDGEMENT}

Authors acknowledge the immense help received from the scholars whose articles are cited and included in references of this manuscript. The authors are also grateful to authors/ editors/publishers of all those articles, journals and books from where the literature for this article has been reviewed and discussed.

\section{Conflict of interest - None}

Financial support - None

Author contribution - AB, RS, SK, and SA conducted research, collected data, analysed and interpreted the data. AW, SA and SB were responsible to look at, design the study and arrange for the research materials. All authors revised the final draft and were involved in each part in finishing the manuscript.

\section{REFERENCES}

1. Fisher VL, Tahrani AA. Cardiac autonomic neuropathy in patients with diabetes mellitus: current perspectives. Diabetes Metab Syndr Obes. 2017;10:419-434.

2. Pathak A, Gupta S, Kumar S, Agrawal S. Evaluation of cardiovascular autonomic nervous functions in diabetics: Study in a rural teaching hospital. J Pract Cardiovasc Sci. 2017;3:150-7

3. Ewing DJ, Clarke BF. Diagnosis and management of diabetic autonomic neuropathy. $\mathrm{Br}$ Med J Clin Res Ed. 1982;285(6346):916-8.

4. Ahire C, Sarode V, Jadhav K, Shreeram V, Gaidhani N. Prevalence of cardiac autonomic neuropathy in short and long-standing type 2 diabetics in western Maharashtra. Int J Basic Appl Med Res. 2014;3:252-9.

5. Dimova R, Tankova T, Guergueltcheva V, Tournev I, Chakarova $\mathrm{N}$, Grozeva $\mathrm{G}$, et al. Risk factors for autonomic and somatic nerve dysfunction in different stages of glucose tolerance. J Diabetes Complications. 2017;31(3):537-43.

6. Ziegler D, Voss A, Rathmann W, Strom A, Perz S, Roden M, et al. Increased prevalence of cardiac autonomic dysfunction at different degrees of glucose intolerance in the general population: the KORA S4 survey. Diabetologia. 2015;58(5):1118-28.

7. Agalya V, Sumathi S. An Assessment of Pain-Free Blood Glucose Level by Noninvasive Methods. Int J Cur Res Rev. 2021; 13 ( 05): 32-35

8. Shiwaku K, Anuurad E, Enkhmaa B, Kitajima K, Yamane Y. Appropriate BMI for Asian populations. Lancet 2004 ;363(9414):1077.

9. Vinik AI, Erbas T, Casellini CM. Diabetic cardiac autonomic neuropathy, inflammation and cardiovascular disease. J Diabetes Investig. 2013;4(1):4-18.

10. Serhiyenko VA, Serhiyenko AA. Cardiac autonomic neuropathy: Risk factors, diagnosis and treatment. World J Diabetes. 2018;9(1):1-24.

11. Pop-Busui R, Evans GW, Gerstein HC, Fonseca V, Fleg JL, Hoogwerf BJ, et al. Effects of cardiac autonomic dysfunction on mortality risk in the Action to Control Cardiovascular Risk in Diabetes (ACCORD) trial. Diabetes Care. 2010;33(7):1578-84.

12. Witte DR, Tesfaye S, Chaturvedi N, Eaton SEM, Kempler P, Fuller JH, et al. Risk factors for cardiac autonomic neuropathy in type 1 diabetes mellitus. Diabetologia. 2005;48(1):164-71. 
Table 1: Baseline characteristics of all the subjects.

\begin{tabular}{|c|c|c|c|c|}
\hline \multicolumn{2}{|c|}{ Parameters } & Case $(\mathbf{n}=\mathbf{2 5 0})$ & Control(n=250) & p-value \\
\hline \multicolumn{2}{|c|}{ Age(years) } & $43.91 \pm 10.83$ & $44.07 \pm 11.27$ & $0.968, \mathrm{NS}$ \\
\hline \multirow[t]{2}{*}{ Gender } & Male & $140(56 \%)$ & $140(56 \%)$ & $1.000, \mathrm{NS}$ \\
\hline & Female & $110(44 \%)$ & $110(44 \%)$ & \\
\hline \multicolumn{2}{|l|}{ BMI } & $23.94 \pm 3.64$ & $22.54 \pm 2.53$ & $<0.0001, \mathrm{~S}$ \\
\hline \multicolumn{2}{|c|}{ W:H Ratio } & $0.92 \pm 0.06$ & $0.88 \pm 0.07$ & $<0.0001, \mathrm{~S}$ \\
\hline \multicolumn{2}{|c|}{ Basal HR(b/min) } & $78.68 \pm 7.8$ & $74.66 \pm 6.72$ & $<0.0001, \mathrm{~S}$ \\
\hline \multicolumn{2}{|c|}{ Basal SBP(mm Hg) } & $126.56 \pm 8.59$ & $125.29 \pm 9.05$ & $0.029, \mathrm{~S}$ \\
\hline \multicolumn{2}{|c|}{ Basal DBP(mm Hg) } & $79.76 \pm 5.82$ & $79.52 \pm 6.51$ & $0.346, \mathrm{NS}$ \\
\hline \multicolumn{2}{|c|}{ Hypertension } & $53(21.20 \%)$ & $42(16.80 \%)$ & $0.250, \mathrm{NS}$ \\
\hline \multicolumn{2}{|c|}{ FBS } & $117.63 \pm 4.57$ & $91.11 \pm 9.2$ & $<0.0001, \mathrm{~S}$ \\
\hline \multicolumn{2}{|c|}{ OGTT } & $161.91 \pm 13.97$ & $119.16 \pm 13$ & $<0.0001, \mathrm{~S}$ \\
\hline \multicolumn{2}{|c|}{ Sr. Total Cholesterol } & $179 \cdot 3 \pm 31.85$ & $163.09 \pm 24.61$ & $<0.0001, \mathrm{~S}$ \\
\hline \multicolumn{2}{|c|}{ Sr. Triglyceride } & $142.91 \pm 44.82$ & $124.61 \pm 33.84$ & $<0.0001, \mathrm{~S}$ \\
\hline \multicolumn{2}{|c|}{ Sr. HDL } & $40.76 \pm 12.5$ & $45.93 \pm 14.67$ & $<0.0001, \mathrm{~S}$ \\
\hline \multicolumn{2}{|l|}{ Sr. LDL } & $109.96 \pm 32$ & $92.23 \pm 25.49$ & $0.0035, \mathrm{~S}$ \\
\hline \multicolumn{2}{|c|}{ Sr. VLDL } & $28.57 \pm 8.94$ & $24.98 \pm 6.75$ & $0.0009, \mathrm{~S}$ \\
\hline
\end{tabular}

S- significant; NS- non-significant

Table 2: Distribution of cardiovascular autonomic tests as per the cut-off values in all subjects

\begin{tabular}{lcccccc}
$\begin{array}{l}\text { Names of cardiovascu- } \\
\text { lar reflex tests }\end{array}$ & Normal & Borderline & Abnormal & Normal & Borderline & Abnormal \\
E: I Ratio & $198(79.2 \%)$ & $39(15.6 \%)$ & $13(5.2 \%)$ & $239(95.6 \%)$ & $9(3.6 \%)$ & $2(0.8 \%)$ \\
Valsalva Ratio & $225(90 \%)$ & $19(7.6 \%)$ & $6(2.4 \%)$ & $244(97.6 \%)$ & $6(2.4 \%)$ & $\mathrm{o}(\mathrm{o} \%)$ \\
30:15 ratio & $221(88.4 \%)$ & $18(7.2 \%)$ & $11(4.4 \%)$ & $241(96.4 \%)$ & $8(3.2 \%)$ & $1(0.4 \%)$ \\
$\begin{array}{l}\text { Postural Hypotension } \\
\text { Effect of Sustained hand } \\
\text { grip on BP }\end{array}$ & $245(98 \%)$ & $5(2 \%)$ & $\mathrm{o}(\mathrm{o} \%)$ & $249(99.6 \%)$ & $1(0.4 \%)$ & $\mathrm{o}(\mathrm{o} \%)$ \\
\hline
\end{tabular}

Table 3: Univariate logistic regression analysis for assessing predictors of CAN.

\begin{tabular}{lllllll} 
Parameters & $\beta$ & S.E. & P-value & Odds ratio & \multicolumn{2}{c}{$95 \%$ C.I. for Odds ratio } \\
Age & & & & & Lower & Upper \\
BMI & -0.014 & 0.015 & $0.321, \mathrm{NS}$ & 0.986 & 0.958 & 1.014 \\
WC:HC & 0.073 & 0.045 & $0.105, \mathrm{NS}$ & 1.076 & 0.985 & 1.175 \\
TC & 2.634 & 2.350 & $0.262, \mathrm{NS}$ & 13.929 & 0.139 & 1393.126 \\
TG & 0.005 & 0.005 & $0.376, \mathrm{NS}$ & 1.005 & 0.994 & 1.015 \\
HDL & 0.002 & 0.004 & $0.648, \mathrm{NS}$ & 1.002 & 0.995 & 1.009 \\
VLDL & -0.006 & 0.011 & $0.617, \mathrm{NS}$ & 0.994 & 0.972 & 1.017 \\
LDL & 0.005 & 0.017 & $0.772, \mathrm{NS}$ & 1.005 & 0.973 & 1.038 \\
Pre diabetes & 0.006 & 0.004 & $0.150, \mathrm{NS}$ & 1.006 & 0.998 & 1.015 \\
& 1.569 & 0.382 & $<.0001, \mathrm{~S}$ & 4.800 & 2.268 & 10.158
\end{tabular}


Table 3: (Continued)

\begin{tabular}{lllllll} 
Parameters & $\beta$ & S.E. & P-value & Odds ratio & \multicolumn{2}{c}{$95 \%$ C.I. for Odds ratio } \\
Gender & & & & Lower & Upper \\
Female & & & & & \\
Male & -0.408 & 0.307 & $0.185, \mathrm{NS}$ & 0.665 & 0.364 & 1.215 \\
Hypertension & 0.157 & 0.376 & $.676, \mathrm{NS}$ & 1.170 & 0.560 & 2.446 \\
\hline
\end{tabular}

$\beta$ (beta)- regression coefficient in the univariate analysis

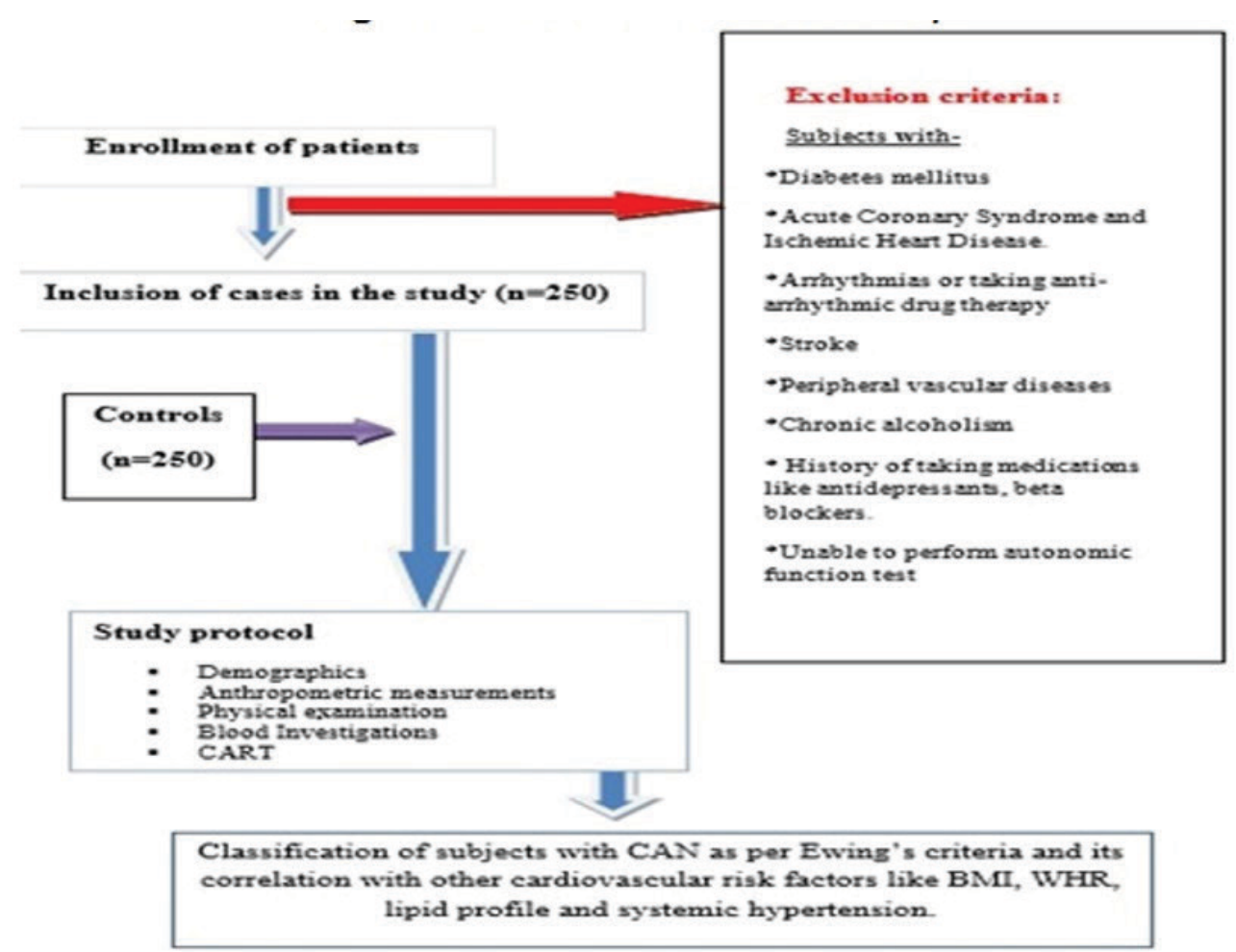

Figure 1: Flow chart of the study.

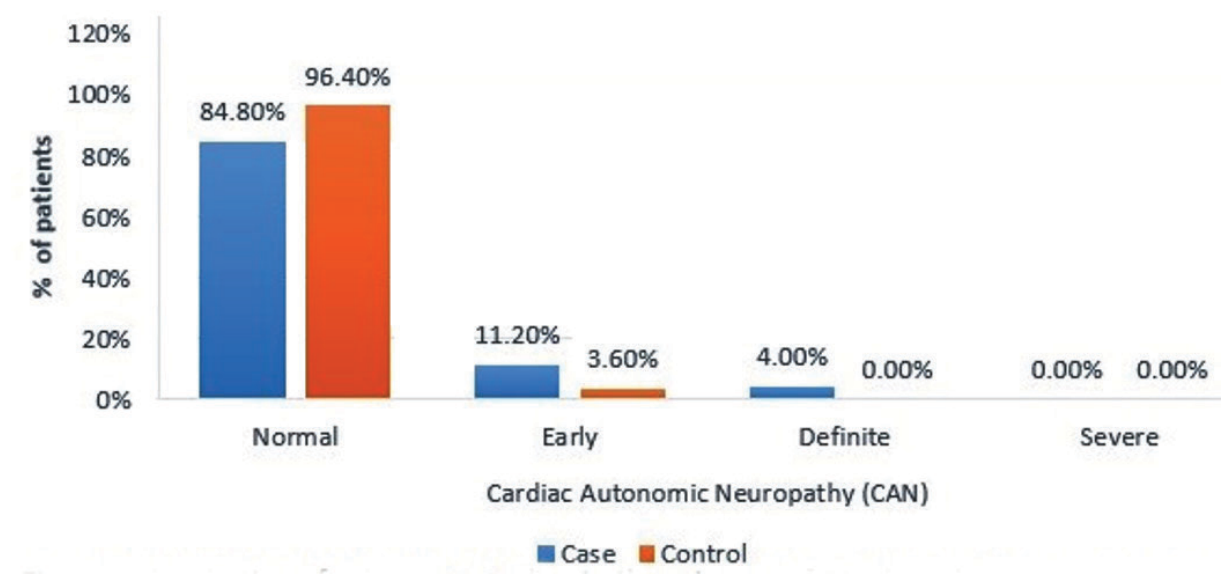

Figure 2: Distribution of subjects according to the category of CAN based on ewings criteria. 